\title{
Impact of the type of anthracycline and of stem cell transplantation in younger patients with acute myeloid leukaemia: Long-term follow up of a phase III study
} \author{
Roelof Willemze $^{5}$ | Sergio Amadori ${ }^{18}$ | Stefan Suciu ${ }^{17}$ \\ ${ }^{1}$ GIGA-I3 and CHU, University of Liège, Liège, Belgium \\ ${ }^{2}$ Gruppo Italiano Malattie Ematologiche Dell'Adulto GIMEMA, Rome, Italy \\ ${ }^{3}$ Radboud University Medical Center, Nijmegen, Netherlands \\ ${ }^{4}$ Sapienza University, Rome, Italy \\ ${ }^{5}$ Leiden University Medical Center, Leiden, Netherlands \\ ${ }^{6}$ Saint-Antoine Hospital, Paris, France \\ ${ }^{7}$ Gemelli Hospital, Rome, Italy \\ ${ }^{8}$ Candiolo Cancer Institute-IRCCS, Torino, Italy \\ ${ }^{9}$ Lyon-Sud University Hospital, Lyon, France \\ ${ }^{10}$ Assistance Publique-Hôpitaux de Paris, Necker Children's Hospital, Paris, France \\ ${ }^{11}$ Universita di Catania, Catania, Italy \\ ${ }^{12}$ Gustave Roussy cancer campus Grand Paris, Villejuif, France \\ ${ }^{13}$ Giovanni XXIII- University Hospital, Bari, Italy \\ ${ }^{14}$ Hospital S. João, Porto, Portugal \\ ${ }^{15}$ University Hospital Centre Zagreb, Zagreb, Croatia \\ ${ }^{16}$ Cardarelli Hospital, Naples, Italy \\ ${ }^{17}$ EORTC Headquarters, Brussels, Belgium \\ ${ }^{18}$ University Tor Vergata, Rome, Italy
}

Frédéric Baron ${ }^{1}$ (1) | Fabio Efficace ${ }^{2}$ ( ) | Laura Cannella ${ }^{2}$ | Petra Muus $^{3}$ | Silvia Trisolini $^{4}$ | Constantijn J. M. Halkes ${ }^{5}$ (1) | Paola Fazi ${ }^{2}$ | Marco Vignetti ${ }^{2}$ | Jean-Pierre Marie $^{6}$ | Patrizia Chiusolo ${ }^{7}$ | Walter van der Velden ${ }^{3}$ (i) | Edoardo La Sala $^{2}$ | Umberto Vitolo ${ }^{8}$ | Xavier Thomas ${ }^{9}$ | Francois Lefrère Sr. ${ }^{10}$ | Francesco Di Raimondo $^{11}$ | Jean-Henri Bourhis ${ }^{12}$ | Giorgina Specchia ${ }^{13}$ | José E. Guimarães ${ }^{14}$ | Bernardino Allione ${ }^{8}$ | Radovan Vrhovac ${ }^{15}$ | Felicetto Ferrara ${ }^{16}$ | Marian Stevens-Kroef ${ }^{3}$ | Liv Meert $^{17}$ | Theo de Witte ${ }^{3}$ |

Correspondence

Frédéric Baron, University of Liège, Department of Hematology, CHU Sart-Tilman 4000 Liège Belgium.

Email: f.baron@ulg.ac.be
Abstract

We provide a long-term evaluation of patients enrolled in the EORTC/GIMEMA AML-10 trial which included a total of 2157 patients, $15-60$ years old, randomized to receive either daunorubicin (DNR, $50 \mathrm{mg} / \mathrm{m}^{2}$ ), mitoxantrone (MXR, $12 \mathrm{mg} / \mathrm{m}^{2}$ ), or idarubicin (IDA, $10 \mathrm{mg} / \mathrm{m}^{2}$ ) in addition to standard-dose cytarabine and etoposide for induction chemotherapy and intermediate dose cytarabine for 
consolidation. Younger patients who reached complete remission with complete (CR) or incomplete (CRi) recovery were then scheduled to receive an allogeneic hematopoietic stem cell transplantation (HSCT). That was if they had a HLAidentical sibling donor; in all other cases, an autologous HSCT had to be administered. At an 11-year median follow-up, the 5-year, 10-year and 15-year overall survival (OS) rates were $33.2 \%, 30.1 \%$ and $28.0 \%$, respectively. No significant difference between the three randomized groups regarding OS was observed $(P=.38)$. In young patients, $15-45$ years old, no treatment difference $(P=.89)$ regarding OS was observed, while in patients $46-60$ years old, MXR and IDA groups had a trend for a longer OS as compared to the DNR group ( $P=.029$ ). Among younger patients without a favorable MRC cytogenetic risk subgroup who achieved a CR/CRi after induction chemotherapy, those with a HLA-identical sibling donor had higher 10-year and 15-year OS rates than those without. In older patients who reached CR/CRi, the long-term outcomes of those with or without a donor was similar. In conclusion, long-term outcomes of the study confirmed similar OS in the three randomized groups in the whole cohort of patients.

\section{1 | INTRODUCTION}

Sixty to $80 \%$ of $15-60$ years old patients with acute myeloid leukemia (AML) achieve a complete remission (CR) after a combination of cytarabine and an anthracycline such as daunorubicin (DNR), mitoxantrone (MXR), or idarubicin (IDA). ${ }^{1}$ In order to compare the relative efficacy of these three anthracyclines, the EORTC and GIMEMA groups conducted a large international phase III randomized controlled trial (RCT) which started to enroll patients in $1993 .{ }^{2}$ With a median follow-up of 5.6 years, main observations were that the use of different anthracyclines had no impact on outcomes in patients with a HLA-identical sibling donor. However, disease-free survival (DFS) and survival from CR were each longer in the MXR and IDA arms than in the DNR arm in the subgroup of patients without a donor. ${ }^{2}$

Still, the best post-remission treatment for younger AML patients remains debated. ${ }^{1,3-5}$ Most prospective studies assessing the impact of having a HLA-identical sibling donor on AML outcomes ("genetic randomization") according to cytogenetic risk have been reported with a relatively short ( $\approx 5-6$ years) median follow-up. ${ }^{6-8}$ This is a significant gap in our knowledge as nonrelapse mortality (NRM) due to complications of chronic graft-vs-host disease may occur well beyond 5 years post transplantation ${ }^{9}$ while late relapses are not infrequent in patients not offered an allogeneic transplantation. ${ }^{10}$

We herein provide results of the very long-term follow-up of patients included in the large EORTC/GIMEMA AML-10 trial. $^{2}$ The primary objective was to assess the long-term impact of the type of anthracycline given in the induction chemotherapy. The secondary objective was to evaluate, in patients who reached a CR/CRi, the difference in the long-term outcome of those who a HLA-identical donor vs those without such a donor. Subgroup analyses by age and cytogenetic features were investigated.

\section{2 | METHODS}

\section{1 | Study design}

Details of the EORTC/GIMEMA AML-10 trial design have been reported in the initial publication. ${ }^{2}$ Briefly, inclusion criteria comprised age 15 to 60 years; diagnosis of primary or secondary AML (including AML occurring after myelodysplastic syndrome) other than French-American-British M3; no evidence of severe concurrent cardiac, pulmonary, neurologic, and metabolic diseases or uncontrolled infections; and adequate liver (serum bilirubin level $<2 x$ upper normal limit) and renal (serum creatinine $<2 \times$ upper normal limit) function tests. Exclusion criteria included blast crisis of chronic myeloid leukemia and AML supervening after other chronic myeloproliferative diseases and other progressive malignant diseases.

Eligile patients were randomized to receive either DNR $\left(50 \mathrm{mg} / \mathrm{m}^{2}\right)$, MXR $\left(12 \mathrm{mg} / \mathrm{m}^{2}\right)$, or IDA $\left(10 \mathrm{mg} / \mathrm{m}^{2}\right)$ on days 1,3 and 5 in addition to standard-dose cytarabine $\left(25 \mathrm{mg} / \mathrm{m}^{2}\right.$ bolus followed by $100 \mathrm{mg} / \mathrm{m}^{2}$ given as a continuous infusion daily for 10 days), and etoposide $\left(100 \mathrm{mg} / \mathrm{m}^{2}\right.$ on days 1-5) for induction chemotherapy. Randomization was stratified according to centre, age (15 to 45 years vs 46 to 60 years), white blood cell count ( $<50$ vs 50 to 249 vs $\geq 250 \times 10^{9} / \mathrm{L}$ ), and WHO performance status ( 0 to 2 vs 3 to 4 ) using a minimization technique.

A second cycle of induction was administered in patients who achieved a partial response (PR). Patients who achieved a CR or a CR with incomplete blood cell counts recovery (CRi) after one or two courses of induction chemotherapy received a consolidation course 
with the same anthracycline as in the induction course. They also received intermediate dose cytarabine $\left(500 \mathrm{mg} / \mathrm{m}^{2}\right.$ every 12 hours as a 2-hour IV infusion on days 1-6). Younger patients, 15 to 45 (or 55, according to centre policy) years old were then scheduled to undergo an allogeneic hematopoietic stem cell transplantation (allo-HSCT) in first CR/CRi if they had a HLA-identical sibling donor. Younger patients without a HLA-identical sibling donor as well as older patients who reached a CR/CRi had to receive an autologous HSCT (auto-HSCT) after consolidation chemotherapy.

Criteria for response and relapse followed the Report of the National Cancer Institute-Sponsored Workshop. ${ }^{11}$ The primary end point was overall survival (OS). Secondary end points included CR/CRi rate after induction, the DFS and OS from CR/CRi and the rate of completion of auto- and allo-HSCT.

\subsection{Ethic statement}

The study was approved by the ethics committees of the participating institutions and was conducted in accordance with the Declaration of Helsinki. All participants gave their informed consent.

\section{3 | Cytogenetic assessment}

Cytogenetic examinations were performed at diagnosis. Cytogenetic data were centrally reported according to International System for Cytogenetic Nomenclature (ISCN) and centrally reviewed. ${ }^{2}$ For the current analysis, cytogenetics were centrally re-reviewed, described according to International System for Cytogenetic Nomenclature (ISCN), ${ }^{12}$ and classified using the refined UK Medical Research Council (MRC) classification ${ }^{13}$ as previously reported. ${ }^{14}$

\section{4 | Statistical analyses}

The duration of OS was calculated from the date of randomization until death, of any cause. The DFS was calculated as the time from CR/CRi until the first relapse or death, whichever occurred first. OS from CR/CRi was calculated as the time from CR/CRi until death.

The Kaplan-Meier method was used to estimate these time-toevent distributions and the Greenwood formula to compute SEs and confidence intervals $(\mathrm{Cl})$ for the 10-year OS, OS and DFS from CR/CRi rates. ${ }^{15}$ Logrank test was used to compare these time-to-event distributions between groups and the Cox proportional hazards model to compute the hazard ratio (HR) and its corresponding $\mathrm{Cl}^{15}$

The estimates of the cumulative incidences of relapse and of death without relapse from the date of CR/CRi, along with their SEs, were based on competing risk methods. ${ }^{16,17}$ For the comparisons of cumulative incidences between groups, and for producing forest plots, the Fine-Gray model was used. ${ }^{18}$

The comparison of the three randomized groups regarding OS was performed at a two-sided significance level of 0.05 , and the two pairwise comparisons (MXR vs DNR and IDA vs DNR) were performed at the 0.025 alpha level. In patients who reached CR/CRi, the time-to-event outcome comparisons of the randomized groups for donor vs no donor, and for subgroup analyses, the tests were performed at a two-sided significance level of 0.01. For these comparisons, the $99 \% \mathrm{Cls}$ of the HRs were provided. Same was done when we combined the two experimental arms (IDA and MXR) - unplanned analyses in the protocol - in order to produce forest plots for OS and OS from CR/CRi. In this setting, a test of interaction between some variables (eg, cytogenetic group or age) and the treatment group (IDA/MXR vs DNR) or donor availability in a Cox model was undertaken. Note, SAS 9.4 (SAS Institute Inc. Cary, NC) was used for all statistical analyses.

\section{3 | RESULTS}

\subsection{Patients}

Between November 1993 and December 1999, a total of 2157 patients were randomly assigned in the trial (Figure S1). ${ }^{2}$ Median age was 44 years (range, 15-60 years). Majority of patients had de novo AML $(n=2064)$, the remaining secondary AML $(n=46)$ or therapy related AML $(n=47)$. The characteristics of the patients were well balanced between the three randomized treatment groups (Table S1). Results were initially published with a median follow-up of 5.6 years. In the current report, median follow-up was 11 years.

\subsection{Impact of the type of anthracycline in the induction/consolidation chemotherapy}

\subsection{1 | Overall survival}

The overall 5-, 10- and 15-year OS rates were $33.2 \%, 30.1 \%$ and $28.0 \%$ respectively. No significant difference between the three randomized groups regarding OS was observed ( $P=.38$; Figure $1 \mathrm{~A}$ ).

We first studied whether there was an impact of age on the association between the type of induction treatment and OS (as mentioned in the material and method section randomization was stratified for patient age: $<46$ vs $\geq 46$ years) insuring the integrity of this analysis. Age impacted (test for interaction: $P=.13$ ) the treatment difference regarding OS (Figure S2A). In young (< 46 years) patients, the OS was comparable in the three treatment groups $(P=.89$; Figure $1 \mathrm{~B}$ ). While OS was shorter in patients $\geq 46$ than $<46$ years old, in older patients OS was prolonged in the MXR and IDA groups, as compared to the DNR group ( $P=.07$ for the three-arm comparison, Figure $1 C$, and $P=.029$, for the comparison between MXR and IDA vs DNR patients combined, HR 0.84, 99\% $\mathrm{Cl}$ 0.69-1.03), Figure S2A). Results remained unchanged by further adjustments by age and MRC cytogenetic risk group (data not shown).

As expected, the initial MRC cytogenetic risk group had an important impact on OS. The 10-year OS rates were $56.5 \%$, 
(A)

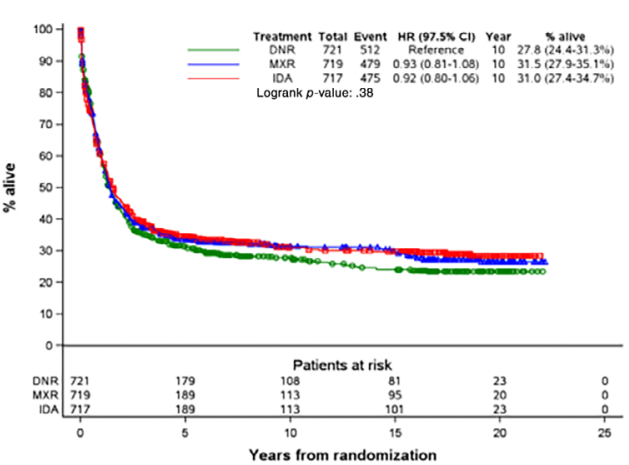

(C)

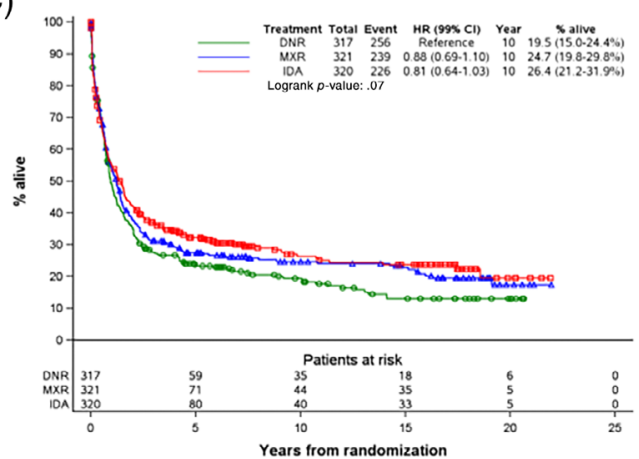

(E)

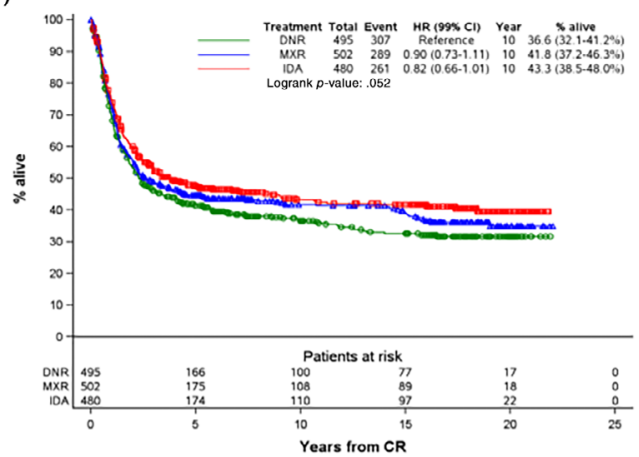

(B)

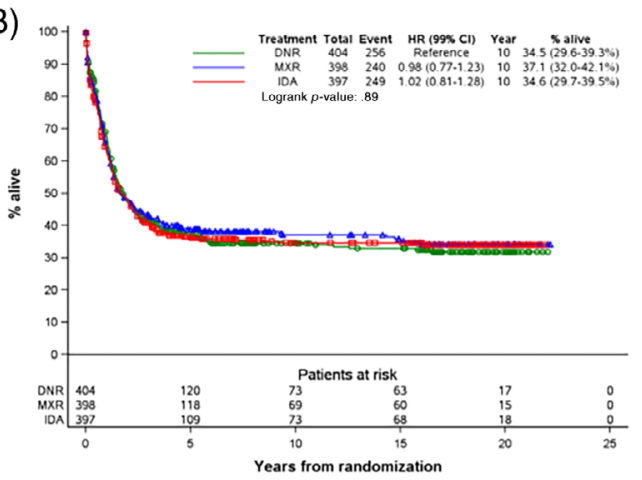

(D)

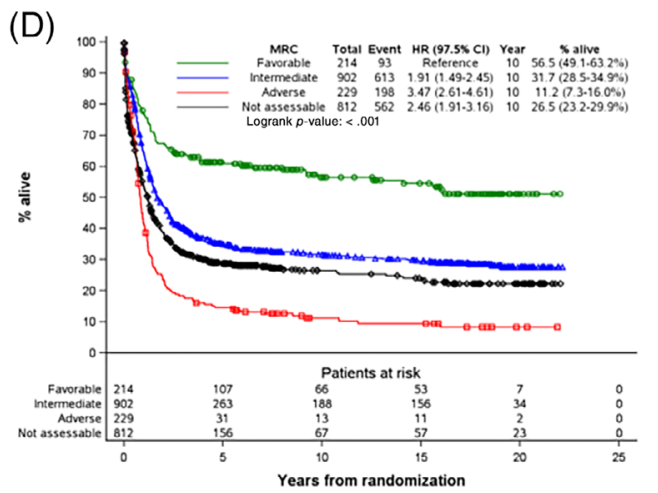

(F)

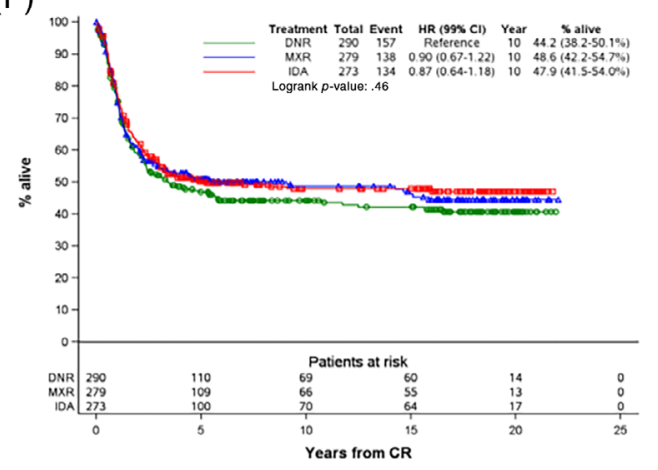

(G)

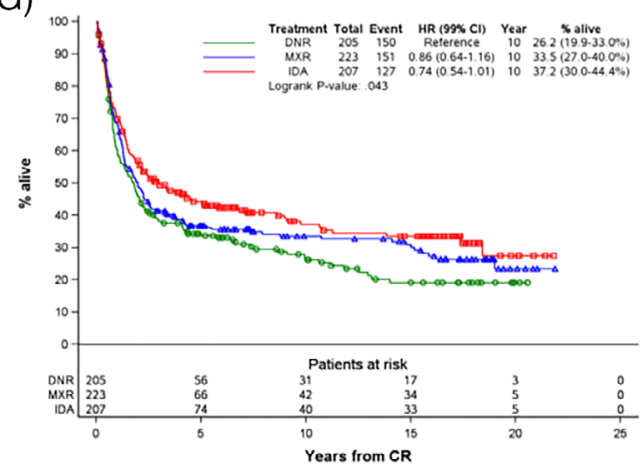

FIGURE 1 Kaplan-Meier plots for A, overall survival (OS) according to the randomized arm in the whole cohort. B, OS according to the randomized arm in patients 15-45 years old. C, OS according to randomized arm in patients 46-60 years old. D, OS according to MRC cytogenetic risk classification. E, OS from CR/CRi in the whole study population. F, OS from CR/CRi according to the randomized arm in patients 15-45 years old. G, OS from CR/CRi according to the randomized arm in patients $46-60$ years old

$31.7 \%$ and $11.2 \%$ in the favorable, intermediate and adverse risk group, respectively (Figure 1D). However, MRC cytogenetic risk group had a limited impact on the treatment difference (test for interaction: $P=.26$; Figure $2 \mathrm{~A}$ ), so within each cytogenetic risk subgroup, treatment outcomes were quite homogeneous (Figure S3A-D). 
(A)

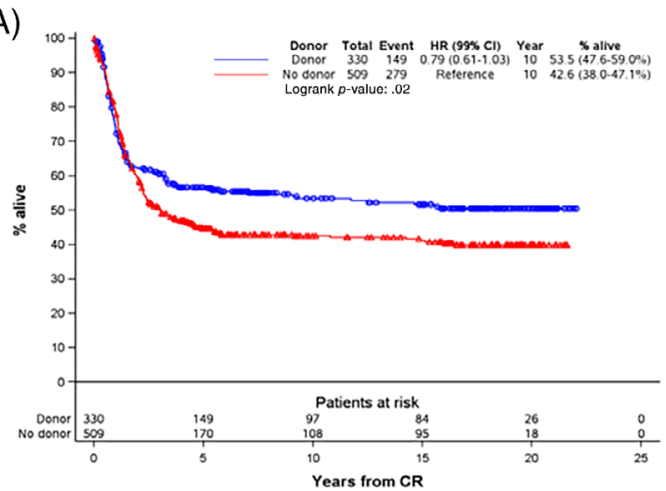

(C)

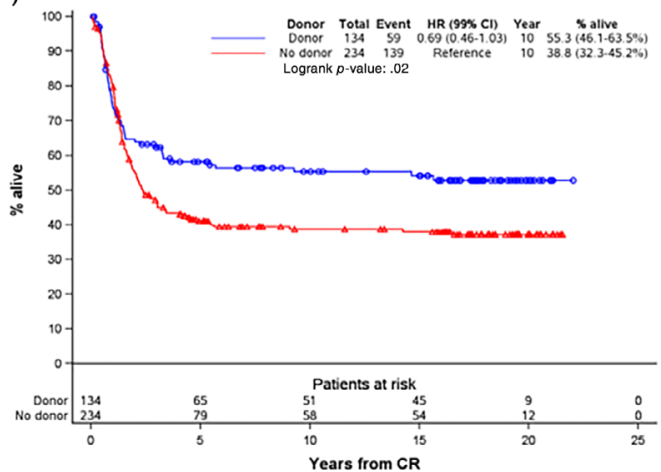

(B)

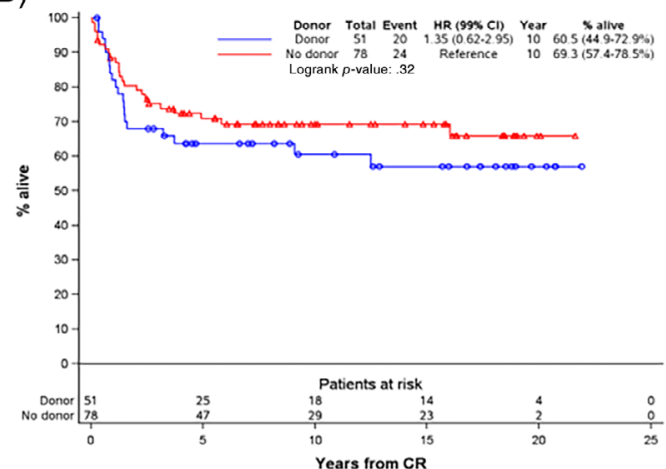

(D)

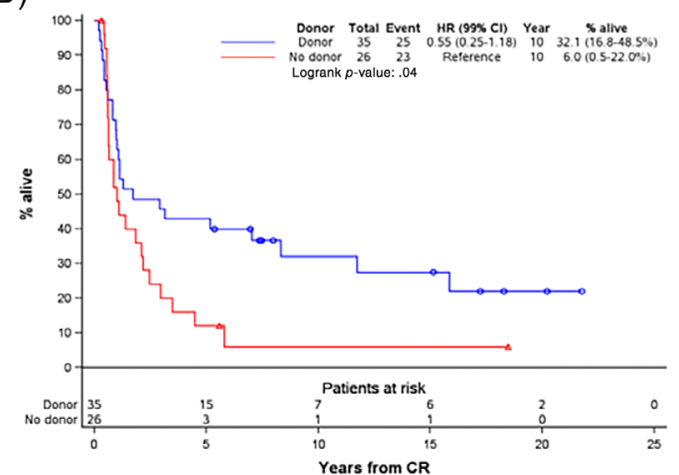

(E)

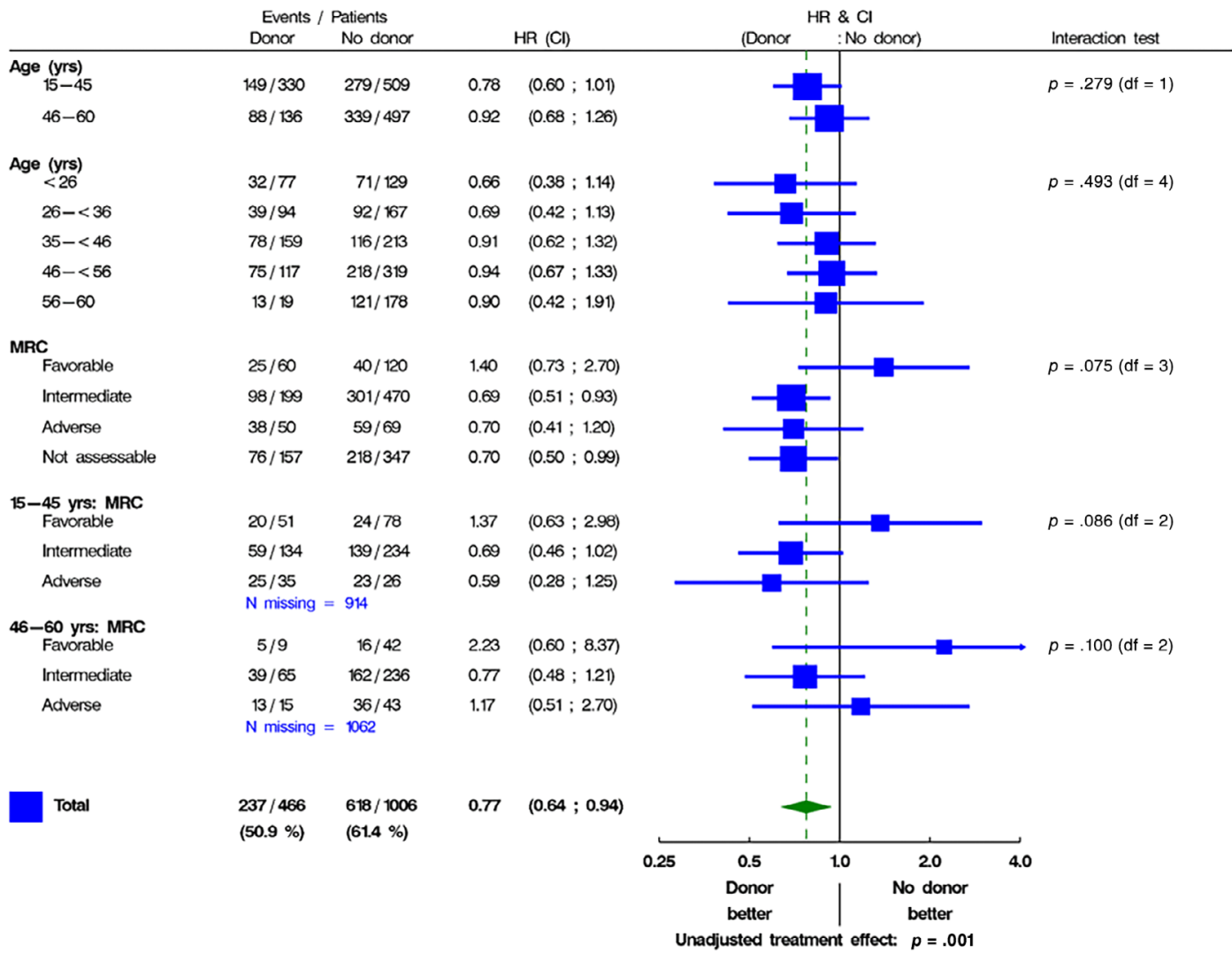

FIGURE 2 Kaplan-Meier plots for overall survival (OS) from CR/CRi according to HLA-identical sibling donor availability A, in patients 15-45 years old. B, in patients 46-60 years old. C, in patients 15-45 years old and MRC favorable cytogenetic risk group; D, in patients 15-45 years old and MRC intermediate cytogenetic risk group; E, Forest plot for OS from CR/CRi according to donor availability: subgroup analysis by MRC cytogenetic risk and age in patients 15-45 years old 


\subsection{2 | Outcomes in patients who reached a CR/CRi}

A CR/CRi after one or two courses of induction chemotherapy was achieved in $69 \%$ of DNR patients, $70 \%$ of MXR patients, and $67 \%$ of IDA patients (Table S2). As expected, patients in the favorable cytogenetic risk group had a higher probability of achieving a CR/CRi (85\%) than those in the intermediate (74\%) or adverse (52\%) MRC cytogenetic risk group.

A HLA-matched sibling donor was found in 466 (32\%) of 1472 typed patients who reached a CR/CRi ( $n=1477$; five patients were not typed). An allo-HSCT was performed in 317 of these 466 patients with a donor (68\%). The allo-HSCT rate in patients with a donor was similar in the three randomized groups. As expected, the allo-HSCT rate was higher in patients $\leq 45$ years $(71 \%)$ than in those
46-60 years old (60\%), Tables 1 and 2. Among 1006 patients without a donor, an auto-HSCT was performed less frequently in patients from the MXR (41\%) or IDA (44\%) arm than in those from the DNR arm (53\%) $(P<.01)$. This was due to higher rate of withdrawals from toxicity and/or lower success rate of sufficient stem cell collection in the MXR and IDA arms in comparison to the DNR arm. ${ }^{2}$ As expected, the auto-HSCT rate was higher in patients $\leq 45$ years (53\%) than in those 46-60 years old (40\%), Tables 1 and 2 . In the latter group, the auto-HSCT rate was $37 \%, 33 \%$ and $49 \%$ in the MXR, IDA and DNR groups, respectively.

The 10-year DFS rates from CR/CRi were similar in the three treatment groups: $31.6 \%$ in DNR patients, $36.9 \%$ in MXR patients, and $36.9 \%$ in IDA patients, respectively $(P=.15)$ (Table S2). There was no significant impact of randomization arm on the relapse incidence or on the incidence of NRM (Table S2), either. Ten-year OS rate from

TAB LE 1 Patient characteristics, treatment applicability and outcomes according to HLA-identical sibling donor availability in patients $<46$ years of age ${ }^{a}$

\begin{tabular}{|c|c|c|c|c|}
\hline & No donor $n=509$ & Donor $n=330$ & & \\
\hline \multicolumn{5}{|l|}{ Age (years), $\mathrm{n}(\%)$} \\
\hline $15-<26$ & $129(25)$ & $77(23)$ & & \\
\hline Assigned HSCT administered, $\mathrm{n}(\%)$ & $267(53)$ & $235(71)$ & & \\
\hline All patients $(n=839)$ & & & $\mathrm{HR}(99 \% \mathrm{Cl})$ & $P$ value \\
\hline 10-year incidence of relapse, \% (SE) & $54.4(2.3)$ & $33.8(2.7)$ & $0.55(0.41-0.74)$ & $<.001^{* *}$ \\
\hline 10-year incidence of nonrelapse mortality, \% (SE\%) & $9.0(1.3)$ & $18.6(2.2)$ & $1.92(1.18-3.12)$ & $<.001^{* *}$ \\
\hline 10-year OS from CR/CRi rate, \% (SE\%) & $42.6(2.3)$ & $53.5(2.9)$ & $0.79(0.61-1.03)$ & $.02^{*}$ \\
\hline \multicolumn{5}{|c|}{ Outcome according to the refined MRC cytogenetic risk group ${ }^{13}$} \\
\hline 10-year DFS from CR/CRi rate, \% (SE\%) & & & HR $(99 \% \mathrm{Cl})$ & $P$ value \\
\hline Adverse $(n=61)$ & $8.1(5.5)$ & $25.4(7.4)$ & $0.62(0.30-1.31)$ & $.096^{*}$ \\
\hline \multicolumn{5}{|l|}{ 10-year cumulative incidence of relapse, \% (SE\%) } \\
\hline Not assessable $(n=281)$ & $57.1(4.4)$ & $33.3(4.6)$ & $0.58(0.34-0.99)$ & $.005^{* *}$ \\
\hline Favorable $(n=129)$ & $29.1(5.2)$ & $24.1(6.1)$ & $0.76(0.31-1.90)$ & $.45^{* *}$ \\
\hline Intermediate $(n=368)$ & $58.6(3.3)$ & $31.9(4.1)$ & $0.44(0.27-0.70)$ & $<.001^{* *}$ \\
\hline Adverse $(n=61)$ & $83.8(7.4)$ & $54.3(8.4)$ & $0.47(0.21-1.05)$ & $.016^{* *}$ \\
\hline \multicolumn{5}{|l|}{ 10-year OS from CR/CRi rate, \% (SE\%) } \\
\hline Not assessable $(n=281)$ & $41.0(4.1)$ & $56.3(4.9)$ & $0.71(0.45-1.14)$ & $.06^{*}$ \\
\hline
\end{tabular}

Note: Forest plots of the hazard ratio by donor availability, according to age and cytogenetic MRC risk group are provided in the supplemental Figures 4A-D (four endpoints: DFS, incidence of relapse, incidence of death without relapse, OS from CR/CRi).

Abbreviations: $\mathrm{Cl}$, confidence interval; $\mathrm{CR}$, complete remission; $\mathrm{CRi}, \mathrm{CR}$ with incomplete blood recovery; DFS, disease-free survival; HSCT, hematopoietic stem cell transplantation; MRC, UK Medical Research Council; N, number; OS, overall survival; SE, standard error; HR, hazard ratio.

anformation missing for three patients.

${ }^{*} P$ value provided by the logrank test.

${ }^{* *} P$ value provided by the Gray test. 
TABLE 2 Patient characteristics, treatment applicability and outcomes according to HLA-identical sibling donor availability in patients 46-60 years of age ${ }^{a}$

\begin{tabular}{|c|c|c|c|c|}
\hline & No donor $N=497$ & Donor $N=136$ & & \\
\hline \multicolumn{5}{|l|}{ Age (years), n (\%) } \\
\hline $46-50$ & $163(33)$ & $71(52)$ & & \\
\hline Assigned HSCT administered, n (\%) & $197(40)$ & $82(60)$ & & \\
\hline All CR/CRi patients $(n=635)$ & & & $\mathrm{HR}(99 \% \mathrm{Cl})$ & $P$ value \\
\hline 10-year incidence of relapse, \% (SE) & $59.0(2.3)$ & $42.7(4.3)$ & $0.66(0.45-0.97)$ & $.003^{* *}$ \\
\hline 10-year nonrelapse mortality, \% (SE\%) & $15.3(1.7)$ & $24.7(3.8)$ & $1.66(1.00-2.75)$ & $.01^{* *}$ \\
\hline 10-year OS from CR/CRi rate, \% (SE\%) & $31.0(2.3)$ & $37.0(4.3)$ & $0.92(0.67-1.25)$ & $.46^{*}$ \\
\hline
\end{tabular}

Note: Forest plots of the hazard ratio by donor availability, according to age and cytogenetic MRC risk group are provided in the supplemental Figures 3A-D (four endpoints: DFS, incidence to relapse, incidence of death without relapse, OS from CR/CRi).

Abbreviations: $\mathrm{Cl}$, confidence interval; CR, complete remission; DFS, disease-free survival; HR, hazard ratio; $\mathrm{HSCT}$, hematopoietic stem cell transplantation; $\mathrm{N}$, number; OS, overall survival; $\mathrm{SE}$, standard error.

anformation missing for two patients.

${ }^{*} P$ value provided by the overall logrank test.

${ }^{* *} P$ value provided by the Gray test.

CR/CRi was $36.7 \%$ in DNR patients, $41.8 \%$ in MXR patients, and 43.3\% in IDA patients, respectively $(P=.052)$ (Table S2).

We also analyzed the impact of age, stratification factor for the randomization, on the association between the type of induction treatment and DFS and OS from CR/CRi. In young ( $\leq 45$ years) patients, the DFS from CR/CRi $(P=.50)$ as well as OS from CR/CRi $(P=.46)$ were comparable in the three treatment groups (Table S2, Figure $1 F)$. In contrast, in older patients, who had globally worse outcomes than younger patients, DFS from CR/CRi $(P=.12)$ and OS from $\mathrm{CR} / \mathrm{CRi}(P=.04)$ were prolonged in the MXR and IDA arms as compared to the DNR arm (Table S2, Figure S2B, Figure 1G).

Results were confirmed in multivariate analysis, where the treatment comparison was adjusted by age and MRC cytogenetic risk group (data not shown).

\subsection{Impact of having a HLA-identical sibling donor in patients who reached a CR/CRi}

\subsection{1 | Patients $\leq 45$ years}

The comparison of outcomes following auto- vs allo-HSCT was first assessed in a cohort of 839 patients $\leq 45$ years of age who achieved a $\mathrm{CR} / \mathrm{CRi}$ after induction chemotherapy, who were HLA-typed, and who mostly were given consolidation chemotherapy. A total of 330 patients had an HLA-identical sibling (donor group) while the remaining 509 patients had not (Table 1). As shown in Figure 2A and in Table 1, the 10-year DFS (HR 0.76, 99\% Cl 0.59-0.97), and OS from CR/CRi (HR 0.79, 99\% Cl 0.61-1.03) rates were approximately $10 \%$ higher in patients with a donor than in those without. This result was due to a lower incidence of relapse (HR 0.55, 99\% Cl 0.41-0.74), and despite an increased incidence of NRM (HR 1.92, 99\% Cl 1.18-3.12) in the donor group (Table 1, forest plots Figure S4). Assessing the impact of donor availability on the outcomes from CR/CRi according to cytogenetic risk group, we observed that patients with a donor had higher 10- and 15- year OS from CR/CRi rates in all but those in the favorable MRC cytogenetic subgroup (Figure 2B-E). Finally, sensitivity analyses using a Cox time-dependent model shows that patients who received allo-HSCT had a longer DFS (HR 0.67, 99\% Cl 0.47-0.95) and OS from HSCT (HR 0.77, 99\% Cl 0.54-1.11), compared to those who received auto-HSCT.

\subsection{2 | Patients $46-60$ years}

The impact between allo-HSCT and auto-HSCT was then assessed in a cohort of 635 patients 46-60 years old, who achieved a $\mathrm{CR} / \mathrm{CRi}$ after induction chemotherapy, and who generally received consolidation chemotherapy. A total of 136 patients had a HLAidentical sibling (donor group) while 497 patients had not (donor availability was unknown in two additional patients) (Table 2). Among patients aged $46-60$ years, 635 reached CR/CRi. The outcomes (DFS and OS) of CR/CRi patients with a donor was only marginally prolonged as compared to those without a donor (Figure 2E). Indeed, the positive effect of decreased relapse incidence (HR 0.66, 99\% $\mathrm{Cl} 0.45-0.97 ; P=.003$ ) was neutralized by an increased risk of death in CR/CRi (HR 1.66, 99\% Cl 1.0-2.75; $P=.013$ ) (Table 2; forest plots in Figure S5). Results remained practically unchanged by adjusting the comparison by age, randomized treatment and MRC cytogenetic risk group (data not shown). Finally, sensitivity analyses using a Cox time-dependent model indicated that similar DFS from HSCT (HR 0.85, 99\% Cl 0.54-1.33), and OS from HSCT (HR 0.95, 99\% $\mathrm{Cl} 0.60-1.51)$ were obtained in allo-HSCT compared to auto-HSCT recipients. 


\section{4 | DISCUSSION}

Here we report the long-term follow-up of one of the largest phase III RCT comparing various anthracyclines in the remission induction and consolidation treatment of AML. Further, we assessed the long-term impact of allo-HSCT (in comparison to auto-HSCT) on outcomes in patients who achieved a CR/CRi, using a "genetic randomization" (ie, having a HLA-identical sibling donor or not).

First, with the long-term follow-up of the study, we confirmed that there was no significant difference between the three randomized groups regarding OS in the whole study population. However, we observed a trend for a higher 10-year OS rate in patients randomized to receive MXR or IDA in the induction and consolidation, as compared to those to receive DNR in the subgroup of patients 46 to 60 years of age. This was not the case in younger patients, possibly because a higher proportion of younger patients received an alloHSCT, offsetting the insufficient efficacy of DNR in this subgroup of patients. Furthermore, in the entire study population, patients randomized in the MXR or IDA arms had also a trend for a higher 10-year OS from CR/CRi rate than those in the DNR arm; the corresponding increase was approximately $7 \%$ and $11 \%$, respectively. These findings were not yet clear when the study was first evaluated at a median follow-up of 5.6 years, stressing the importance of reporting longterm follow up of large RCT. Even at long-term follow-up our study confirms the superiority of IDA over DNR regarding OS as reported by a Cochrane meta-analysis of RCTs reported in 2015 including data from 9549 patients. ${ }^{19}$ The lower 10 -year OS rate observed with DNR could be due to the fact that the dose of DNR used in the AML-10 trial (as well as in several trials included in the above-mentioned metaanalysis) was suboptimal (DNR/IDA ratio of 5). Note, a recent phase III trial observed similar outcomes in AML younger patients randomized to receive induction with cytarabine and high-dose DNR $(90 \mathrm{mg} /$ $\mathrm{m}^{2} \times 3$ days) or IDA (12 mg/ $\mathrm{m}^{2} \times 3$ days). ${ }^{20}$ In addition, superior outcomes with higher than standard doses of DNR ( 90 vs $45 \mathrm{mg} / \mathrm{m}^{2}$ ) were reported by the ECOG. ${ }^{21}$ Interestingly, a meta-analysis of RCT performed in children, and adults $<60$ years of age $(n=3382)$ reported in 2013 that the superiority of IDA for remission induction chemotherapy was restricted to RCT with a DNR/IDA ratio $<5 .{ }^{22}$ More recently, the UK-NCRI group reported similar OS but higher 60-day mortality with DNR $90 \mathrm{mg} / \mathrm{m}^{2}$ in comparison to DNR $60 \mathrm{mg} / \mathrm{m}^{2}{ }^{23}$

Another observation of our study was that the feasibility of performing an auto-HCT was higher in DNR than in IDA or MXR patients. Interestingly, this did not translate to lower relapse risk in DNR patients despite prior RCT from our group that demonstrated better outcomes with auto-HCT than with additional chemotherapy. ${ }^{24}$

Late NRMs (due in a large part to complications of chronic graftvs-host disease) are not infrequent beyond 5 years post allo-HSCT, ${ }^{9}$ while more late relapses can occur in patients not offered an alloHSCT. ${ }^{10,24}$ In fact, a recent study by the European Society for Blood and Marrow Transplantation has demonstrated that, among AML patients who were disease free at least for 2 years after an autoHSCT, the 5- and 10-year incidences of relapse from auto-HSCT were $11 \%$ and $16 \%$, respectively. ${ }^{25}$ Consequently, long-term follow-up of studies assessing the impact of post-remission treatment with alloHSCT are of major interest. In studies where information on the donor availability at the achievement of CR/CRi is not collected, the MantelByar method ${ }^{26}$ or Cox time-dependent model should be used. This is to avoid the guarantee time bias in the outcome comparison of patients who received allo-HSCT, vs the other CR/CRi patients. In order to eliminate the bias of such comparison due to patients' selection, which is stricter for patients allografted, a prospective collection of donor availability in CR/CRi patients is mandatory in wellconducted studies, like RCT. The comparison based on the availability HLA-match donor vs no donor ("genetic randomization") provides an unbiased assessment of the allo-HSCT outcome, so it became the gold standard statistical method in this setting. ${ }^{27}$ Here, we observed that, in the group of patients $<46$ years of age, those with a HLA-identical sibling donor ( $71 \%$ of them received an allo-HSCT) had a lower risk of relapse translating to higher 10 -year and 15 - year DFS and OS rates from CR/CRi in all patients but those with favorable risk cytogenetics. This occurred despite a higher NRM in patients with a donor. Interestingly, the advantage of having a donor in the subgroup of patients with intermediate-risk cytogenetics was not apparent at the time of the initial report, ${ }^{6}$ further stressing the importance of long-term follow-up studies.

While transplant-related mortality has dramatically decreased in the last decades, one could argue on the continued relevance of our donor vs no donor analyses. On the other hand, given the progresses in alternative donor transplantation, the vast majority of patients have nowadays a suitable donor. Consequently, studies comparing alloHSCT to auto-HSCT and/or chemotherapy using a donor vs no donor genetic randomization are no longer feasible. Since reduction of NRM will result in a higher benefit of having a donor, the message of our paper that having a donor (and thus receiving an allo-HSCT) is beneficial to patients with intermediate and adverse cytogenetic remains true for transplanted patients. It should be however noted that the decision of allo-HCT or not in patients with intermediate-risk cytogenetic is mainly based on FLT3-ITD and NPM1 status of leukemia, as well as on persistence of not or measurable residual disease.

In patients 46 years of age or older, there was no overall benefit of having a donor because the reduction of relapse in this group of patients was offset by a higher NRM. Further, even within the subgroup of patients $<46$ years old, the advantage of having a donor was more pronounced in very younger patients (15 to 29 years) than in those 30 to 45 years old. These observations might be due to the fact that AML-10 trial was performed 20 years ago, before the development of modern reduced intensity/toxicity or truly nonmyeloablative conditioning regimen for transplantation. ${ }^{28}$ Further studies are needed to assess the long-term impact of reduced intensity allo-HSCT in older $\mathrm{AML}$ patients with intermediate or poor risk cytogenetic features. Indeed, recent data have observed that post-remission treatment with a nonmyeloablative allo-HSCT might prolong OS in AML patients even above 60 years of age. ${ }^{29}$

In summary, to the best of our knowledge this study reports the first evidence-based data with regard to long-term impact of anthracycline-type and donor availability on outcomes in younger 
AML patients. Specifically, long-term follow-up of the EORTC/ GIMEMA AML-10 trial confirmed that, overall, similar outcomes were obtained by using either DNR, MXR or IDA (at the studied dosages) during induction and consolidation. In 46-60 years old patients who reached $\mathrm{CR} / \mathrm{CRi}$, having a donor or not yielded similar outcomes; however, MXR and IDA tended to provide better results than DNR given at $50 \mathrm{mg} / \mathrm{m}^{2}$. In addition, in the group of younger patients without favorable cytogenetic features who reached CR/CRi, the longterm outcome was improved when an HLA-identical sibling donor was available.

\section{ACKNOWLEDGEMENTS}

The authors thank all the EORTC Leukemia Group members and the clinicians who participated in this study.

This publication was supported by a donation from the "Fondation contre le cancer"from "Belgium" through the EORTC Cancer Research Fund. FB is senior research associate of the Fund for Scientific Research (FRS-FNRS) Belgium.

\section{CONFLICT OF INTEREST}

Frédéric Baron has received travel grants from Celgene, Abbvie, Novartis and Sanofi. Sergio Amadori has consulting or advisory role for Novartis, Celgene and Abbvie. Theo de Witte received support from Novartis and Celgene. Xavier Thomas received fees for participation to boards with Amgen, Pfizer, Celgene, Abbvie, and Sunesis. Fabio Efficace received consultancy fee from Seattle Genetics, Bristol-Myers Squibb, TEVA, Orsenix and Incyte and research funding from Lundbeck, TEVA and Amgen. The other authors have nothing to disclose with respect to this manuscript.

\section{AUTHOR CONTRIBUTIONS}

F.B, F.E and S.S wrote the manuscript. All authors (FB, FE, LC, PM, ST, C..J..M.H, P..F, M.V, J.P.M, P.C, WvdV, E.L, U.V, X.T, F.L, F.D.R, J.H..B, G..S, J.E.G, B.A, R.V, F.F, M.S.K, L.M, T.dW, R.W, S.A, S.S) participated in study conception and design, data analysis, collection and/or data interpretation and manuscript revising/editing. All authors approved the manuscript and agreed to be accountable for all aspect of the work.

\section{ORCID}

Frédéric Baron (iD) https://orcid.org/0000-0002-2944-3812 Fabio Efficace (D) https://orcid.org/0000-0002-5065-5166 Constantijn J. M. Halkes (D) https://orcid.org/0000-0001-6573-0045 Walter van der Velden (D) https://orcid.org/0000-0002-7002-9701

\section{REFERENCES}

1. Dohner H, Estey EH, Amadori S, et al. Diagnosis and management of acute myeloid leukemia in adults: recommendations from an international expert panel, on behalf of the European LeukemiaNet. Blood. 2010;115(3):453-474.

2. Mandelli F, Vignetti M, Suciu S, et al. Daunorubicin versus mitoxantrone versus idarubicin as induction and consolidation chemotherapy for adults with acute myeloid leukemia: the EORTC and GIMEMA Groups Study AML-10. J Clin Oncol. 2009;27(32):5397-5403.
3. Cornelissen JJ, Blaise D. Hematopoietic stem cell transplantation for patients with AML in first complete remission. Blood. 2016;127 (1):62-70.

4. Gale RP, Wiernik PH, Lazarus HM. Should persons with acute myeloid leukemia have a transplant in first remission? Leukemia. 2014;28(10): 1949-1952.

5. Versluis J, In 't Hout FE, Devillier R, et al. Comparative value of postremission treatment in cytogenetically normal AML subclassified by NPM1 and FLT3-ITD allelic ratio. Leukemia. 2017;31(1):26-33.

6. Suciu S, Mandelli F, de Witte T, et al. Allogeneic compared with autologous stem cell transplantation in the treatment of patients younger than 46 years with acute myeloid leukemia (AML) in first complete remission (CR1): an intention-to-treat analysis of the EORTC/GIMEMAAML-10 trial. Blood. 2003;102(4):1232-1240.

7. Cornelissen JJ, van Putten WL, Verdonck LF, et al. Results of a HOVON/SAKK donor versus no-donor analysis of myeloablative HLA-identical sibling stem cell transplantation in first remission acute myeloid leukemia in young and middle-aged adults: benefits for whom? Blood. 2007;109(9):3658-3666.

8. Burnett AK, Wheatley K, Goldstone AH, et al. The value of allogeneic bone marrow transplant in patients with acute myeloid leukaemia at differing risk of relapse: results of the UKMRC AML 10 trial. $\mathrm{Br}$ J Haematol. 2002;118(2):385-400.

9. Wingard JR, Majhail NS, Brazauskas R, et al. Long-term survival and late deaths after allogeneic hematopoietic cell transplantation. J Clin Oncol. 2011;29(16):2230-2239.

10. Beguin Y, Sautois B, Forget P, Bury J, Fillet G. Long term follow-up of patients with acute myelogenous leukemia who received the daunorubicin, vincristine, and cytosine arabinoside regimen. Cancer. 1997;79(7): 1351-1354.

11. Cheson BD, Cassileth PA, Head DR, et al. Report of the National Cancer Institute-sponsored workshop on definitions of diagnosis and response in acute myeloid leukemia. J Clin Oncol. 1990;8(5):813-819.

12. Simons A, Shaffer LG, Hastings RJ. Cytogenetic nomenclature: changes in the ISCN 2013 compared to the 2009 edition. Cytogenet Genome Res. 2013;141(1):1-6.

13. Grimwade D, Hills RK, Moorman AV, et al. Refinement of cytogenetic classification in acute myeloid leukemia: determination of prognostic significance of rare recurring chromosomal abnormalities among 5876 younger adult patients treated in the United Kingdom Medical Research Council trials. Blood. 2010;116(3):354-365.

14. Baron F, Stevens-Kroef M, Kicinski M, et al. Impact of induction regimen and of allogeneic hematopoietic cell transplantation on outcome in younger adults patients with acute myeloid leukemia with a monosomal karyotype. Haematologica. 2019;104(6):1168-1175.

15. Kalbfleisch JD, Prentice RL. The Statistical Analysis of Failure Time Data. 2nd ed. Hoboken, NJ: John Wiley; 2002.

16. Aalen $\mathrm{OO}$, Johansen $\mathrm{S}$. An empirical transition matrix for nonhomogeneous markov chains based on censored observations. Scand J Stat. 1978;5(3):141.

17. Andersen PK, Borgan O, Gill RD, Keiding N. Statistical Models Based on Counting Processes. New York, NY: Springer-Verlag; 1993.

18. Fine J, Gray RA. A proportional hazards model for the subdistribution of a competing risk. J Am Stat. 1999;99(446):496-509.

19. Li X, Xu S, Tan Y, Chen J. The effects of idarubicin versus other anthracyclines for induction therapy of patients with newly diagnosed leukaemia. Cochrane Database Syst Rev. 2015;6:CD010432.

20. Lee JH, Kim H, Joo YD, et al. Prospective randomized comparison of idarubicin and high-dose daunorubicin in induction chemotherapy for newly diagnosed acute myeloid leukemia. J Clin Oncol. 2017;35(24): 2754-2763.

21. Fernandez HF, Sun Z, Yao X, et al. Anthracycline dose intensification in acute myeloid leukemia. N Engl J Med. 2009;361(13):1249-1259.

22. Teuffel $O$, Leibundgut $K$, Lehrnbecher T, Alonzo TA, Beyene J, Sung L. Anthracyclines during induction therapy in acute myeloid leukaemia: a 
systematic review and meta-analysis. Br J Haematol. 2013;161(2): 192-203.

23. Burnett $\mathrm{AK}$, Russell $\mathrm{NH}$, Hills $\mathrm{RK}$, et al. A randomized comparison of daunorubicin $90 \mathrm{mg} / \mathrm{m}^{2}$ vs $60 \mathrm{mg} / \mathrm{m}^{2}$ in AML induction: results from the UKNCRI AML17 trial in 1206 patients. Blood. 2015;125(25): 3878-3885.

24. Baron F, Efficace F, Cannella L, et al. Long-term follow-up of a trial comparing post-remission treatment with autologous or allogeneic bone marrow transplantation or intensive chemotherapy in younger acute myeloid leukemia patients. Haematologica. 2020;105(1): e13-e16.

25. Czerw T, Labopin M, Gorin NC, et al. Long-term follow-up of patients with acute myeloid leukemia surviving and free of disease recurrence for at least 2 years after autologous stem cell transplantation: a report from the Acute Leukemia Working Party of the European Society for Blood and Marrow Transplantation. Cancer. 2016;122 (12):1880-1887.

26. Mantel N, Byar DP. Evaluation of response-time data involving transient states: an illustration using heart-transplant data. J Am Stat Assoc. 1974;69:81-86.

27. Gray R, Wheatley K. How to avoid bias when comparing bone marrow transplantation with chemotherapy. Bone Marrow Transplant. 1991;7(suppl 3):9-12.
28. Baron F, Storb R. Hematopoietic cell transplantation after reducedintensity conditioning for older adults with acute myeloid leukemia in complete remission. Curr Opin Hematol. 2007;14(2):145-151.

29. Versluis J, Hazenberg CL, Passweg JR, et al. Post-remission treatment with allogeneic stem cell transplantation in patients aged 60 years and older with acute myeloid leukaemia: a time-dependent analysis. Lancet Haematol. 2015;2(10):e427-e436.

\section{SUPPORTING INFORMATION}

Additional supporting information may be found online in the Supporting Information section at the end of this article.

How to cite this article: Baron F, Efficace F, Cannella L, et al. Impact of the type of anthracycline and of stem cell transplantation in younger patients with acute myeloid leukaemia: Long-term follow up of a phase III study. Am J Hematol. 2020;95:749-758. https://doi.org/10.1002/ajh. $\underline{25795}$ 